\title{
SIMULAÇÃO DEM DO EMPACOTAMENTO DE SEMENTES DE ACEROLA
}

\author{
B. S. A. ARAÚJO ${ }^{1}$, M. C. C. FRANCISQUETTI ${ }^{2}$, M. A. S. BARROZO², \\ K. G. SANTOS ${ }^{1}$ \\ ${ }^{1}$ Universidade Federal do Triângulo Mineiro, Departamento de Engenharia Química \\ ${ }^{2}$ Universidade Federal de Uberlândia, Faculdade de Engenharia Química \\ E-mail para contato: brunasenearaujo@gmail.com
}

\begin{abstract}
RESUMO - Neste trabalho, investigou-se os efeitos dos parâmetros do Modelo de Hertz-Mindlin com JKR sobre o comportamento da porosidade de um leito cônico contendo partículas de sementes de acerola, através da técnica de superfície de resposta, a partir de um Planejamento Composto Central (PCC). O empacotamento foi reproduzido utilizando-se de simulações computacionais empregando simulação DEM. O valor experimental de porosidade foi comparado com as simulações e foi possível encontrar o conjunto de parâmetros que melhor representa o comportamento experimental do empacotamento.
\end{abstract}

\section{INTRODUÇÃO}

No Brasil são gerados, anualmente, milhões de toneladas de resíduos decorrentes das atividades agroindustriais como, por exemplo, materiais derivados de processamentos de alimentos, fibras ou madeira. Estes rejeitos representam um potencial poluidor quando dispostos inadequadamente na natureza, pois mesmo sendo biodegradáveis, o volume excessivo e o alto teor de umidade dificultam o processo natural de degradação. Segundo Bortolotti et al. (2013), os resíduos do processamento de acerola representam cerca de $40 \%$ do volume de produção, os quais geralmente são desprezados quando poderiam ser reaproveitados como fontes alternativas de nutrientes.

A secagem é um processo que se realizado sob condições adequadas pode proporcionar o aproveitamento desses resíduos como suplemento alimentar, pois além de remover a umidade do material garantindo seu estado de conservação, ela pode manter a concentração de nutrientes. O leito de jorro é um dos equipamentos mais apropriados para a secagem de grãos e pastas ou suspensões, principalmente por manter um movimento cíclico e homogêneo das partículas, garantindo um eficiente contato fluido-partícula que garante altos coeficientes convectivos de calor e de massa entre as fases.

No entanto, o leito de jorro é um equipamento que apresenta uma fluidodinâmica altamente influenciada pela porosidade do leito estático, sendo fundamental conhecer a porosidade antes de realizar a modelagem e simulação do leito de jorro. Uma possibilidade de prever a porosidade das partículas é a simulação empregando o Método de Elementos Discretos, como realizado por Yi et al. (2011) ao simular o empacotamento de leitos fixos com esferas. 
O Método de Elementos Discretos (DEM) se tornou uma poderosa ferramenta para as indústrias que trabalham com material granulado devido à sua dinâmica, que contabiliza as propriedades físicas e mecânicas dos materiais, tais como, atrito de rolamento e deslizamento. Sua aplicação tem sido estendida à modelagem de sistemas particulados densos, pois contabiliza o efeito do tamanho e da forma real da partícula, além de permitir uma modelagem detalhada da colisão entre as partículas com outras partículas e com a parede do recipiente.

Neste trabalho, foi realizada a simulação do empacotamento de sementes de acerola em uma base cônica, similar a um leito de jorro, pelo Método de Elementos Discretos (DEM) utilizando o software EDEM 2.3, a qual foi comparada aos resultados experimentais obtidos.

\section{O MÉTODO DE ELEMENTOS DISCRETOS}

O Método de Elementos Discretos (DEM) envolve a descrição do movimento de cada partícula no escoamento e a modelagem da colisão entre as partículas e os contornos rígidos, sendo o método mais adequado para lidar com problemas de natureza descontínua, e ao mesmo tempo com grandes níveis de deformação, como é o caso do escoamento granular.

A metodologia DEM e suas variantes estão bem estabelecidas e são descritas em detalhes por Cleary (2008). O modelo de contato, usado para contabilizar as forças de interação entre duas esferas rígidas que pertencem a duas diferentes partículas, é baseado no modelo de Hertz-Mindlin, que caracteriza as interações por meio de três coeficientes: coeficiente de restituição, coeficiente de fricção estático e coeficiente de fricção de rolamento.

No modelo de colisão de Hertz-Mindlin, a componente normal da força é baseada na teoria de contato de Hertz (1882), enquanto a força tangencial é baseada no trabalho de Mindlin (1949). Tanto no cálculo da força normal, quanto da tangencial, há um termo de amortecimento do sistema, relacionado ao coeficiente de restituição, que relaciona a velocidade de separação e a velocidade de aproximação de uma ou mais partículas em uma colisão. No modelo coesivo JKR, a força de fricção depende da parte repulsiva positiva da força normal. Como resultado, o modelo de atrito JKR fornece maiores forças de atrito quando a componente de coesão está presente. Maiores detalhes da modelagem podem ser encontrados em Santos et al. (2014).

\section{METODOLOGIA}

\subsection{Procedimento Experimental}

As sementes de acerola utilizadas no experimento foram provenientes de uma indústria de processamento de alimentos, localizada em Minas Gerais. O tamanho de partícula $\left(d_{p}\right)$ e a densidade aparente foram calculados pelo método de picnometria utilizando o éter de petróleo. A esfericidade foi medida utilizando o HAVER CPA 3-2, um analisador de tamanho de partícula. A Tabela 1 descreve as propriedades do material. 
As massas das partículas foram pesadas e acrescentadas aleatoriamente a um recipiente cônico, similar ao cone do leito de jorro empregado na secagem, até que fosse atingida a altura de leito estático de $0,08 \mathrm{~m}$. Com os dados da densidade aparente e a massa do material, foi possível calcular o valor experimental da porosidade do leito, que corresponde à fração de vazios.

Tabela 1 - Propriedades das partículas de resíduo de acerola

\begin{tabular}{lll}
\hline $\mathrm{d}_{\mathrm{p}}[\mathrm{mm}]$ & Diâmetro de Partícula & $7,83 \pm 0,13$ \\
$\rho_{\mathrm{ap}}\left[\mathrm{kg} / \mathrm{m}^{3}\right]$ & Densidade Aparente & $738 \pm 28$ \\
$\rho_{\text {real }}\left[\mathrm{kg} / \mathrm{m}^{3}\right]$ & Densidade Real & $1344,85 \pm 13,86$ \\
$\phi$ & Esfericidade & $0,68 \pm 0,02$ \\
\hline
\end{tabular}

\subsection{Procedimento de Simulação}

O empacotamento das sementes de acerola em um cone similar a um leito jorro foi reproduzido utilizando-se de simulações computacionais empregando o DEM, implementado no software EDEM 2.3. O processo de empacotamento do leito consistiu em encher o cone progressivamente, gerando partículas a partir de uma superfície virtual na parte superior do leito. As partículas caiam pelo efeito da gravidade dentro do cone até que a altura de leito estático fosse atingida (BORTOLOTTI et al, 2013).

O comportamento de materiais granulares é fortemente dependente do tamanho e da forma da partícula, requerendo uma representação realística da mesma. Nas simulações realizadas neste estudo, a forma do resíduo de acerola foi aproximada pelo método multiesfera, representado na Figura 1. Cada partícula foi gerada por doze esferas, assim o volume médio da partícula de acerola foi 3,24 $10^{-7} \mathrm{~m}^{3}$. O modelo de Hertz-Mindlin com JKR, descrito anteriormente, foi empregado para representar a força de contato entre as partículas de acerola. O passo de tempo em todas as simulações foi fixado como sendo $20 \%$ do passo de tempo crítico calculado pelo tempo de Rayleigh (SANTOS et al., 2014)

Figura 1 - Forma das partículas de resíduo de acerola (método multiesfera).
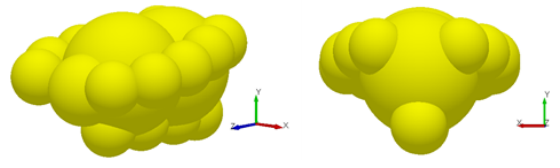

Com o intuito de avaliar os efeitos dos parâmetros do modelo na porosidade, as condições de simulação foram escolhidas a partir de um planejamento composto central (PCC) com cinco variáveis e uma réplica no nível central. As variáveis analisadas foram o módulo de cisalhamento, o coeficiente de restituição, o coeficiente de atrito estático, o coeficiente de atrito de rolamento e a energia de interface (coesão), codificadas de acordo com a Tabela 2. Desta forma, foi possível avaliar quais parâmetros mais influenciaram a simulação, obter o modelo e as superfícies de resposta e concluir qual simulação forneceu um resultado mais próximo do experimental. 
Como na base do leito a relação entre o diâmetro da partícula e o diâmetro do tronco de cone é baixa, há indícios de que ocorrerão problemas de efeito de parede, o que irá aumentar a porosidade nessa região. Assim, para avaliar a distribuição de porosidade ao longo da altura do leito, devido a efeitos de parede, foram realizadas simulações com diferentes alturas de leito estático.

Tabela 2 - Níveis e faixa de valores das variáveis codificadas

\begin{tabular}{llccccc}
\cline { 3 - 7 } & & \multicolumn{5}{c}{ Níveis } \\
\hline \multicolumn{1}{c}{ Variáveis } & $-1,607$ & $-1,000$ & 0,000 & $+1,000$ & $+1,607$ \\
\hline X1 & Módulo Cisalhante [Pa] & $1,17810^{6}$ & $3,00010^{6}$ & $6,00010^{6}$ & $9,00010^{6}$ & $1,08210^{7}$ \\
X2 & Coeficiente de Restituição & 0,018 & 0,200 & 0,500 & 0,800 & 0,982 \\
X3 & Coeficiente de Atrito Estático & 0,018 & 0,200 & 0,500 & 0,800 & 0,982 \\
X4 & Coeficiente de Atrito de Rolamento & 0,018 & 0,200 & 0,500 & 0,800 & 0,982 \\
X5 & Energia de Superfície [J/m $\left./ \mathrm{m}^{2}\right]$ & 0,002 & 0,024 & 0,060 & 0,096 & 0,118 \\
\hline
\end{tabular}

\section{RESULTADOS}

Neste trabalho, os efeitos dos parâmetros do Modelo de Hertz-Mindlin com JKR sobre o comportamento da porosidade de um leito cônico contendo partículas de sementes de acerola foram investigados por meio da técnica de superfície de resposta, a partir de um PCC, disposto na Tabela 3, na qual também se encontram os resultados de porosidade obtidos nas simulações DEM $\left(\varepsilon_{D E M}\right)$ e o desvio $\bar{\sigma}($ em \%) entre o valor experimental, que foi de 0,548 , e o simulado.

A partir dos dados da Tabela 3, pode-se observar que a porosidade simulada varia significativamente de uma simulação para a outra, compreendendo valores entre 0,466 a 0,594. Uma análise de regressão múltipla foi realizada para quantificar o efeito dos parâmetros do modelo de forças de contato sobre a porosidade do leito. A fim de obter a equação que descreve a resposta em função das varáveis independentes foi realizado um teste de hipóteses usando a estatística $t$ de Student para identificar os parâmetros significativos. Os coeficientes da equação estimados pelo método de mínimos quadrados com nível de significância maior que $5 \%,(\mathrm{p}>0,05)$ foram negligenciados. A equação de ajuste para a porosidade simulada $\left(\varepsilon_{D E M}\right)$, é dada por $\left(\mathrm{R}^{2}=0,959\right)$ :

$$
\varepsilon_{D E M}=0,5598-0,0113 X_{2}+0,0264 X_{3}+0,0158 X_{4}+0,0068 X_{5}-0,0157 X_{3}^{2}
$$

A Equação 1 mostra que somente a variável $\mathrm{X}_{1}$, correspondente ao Módulo Cisalhante, não influencia significativamente o empacotamento. A variável que mais influencia $\mathrm{o}$ empacotamento é o coeficiente de atrito estático, que aparece tanto na forma linear quanto na quadrática, possivelmente por que o sistema apresenta baixas velocidades das partículas, como também mostra a Figura 2a). Observou-se que o aumento da Energia de Superfície proporciona um aumento da porosidade do material, como mostra a Figura $2 b$ ), devido à maior coesão que dificulta o deslizamento das partículas, influenciando o mecanismo de empacotamento. Nenhuma das interações entre as variáveis mostrou significativa influência sobre a porosidade do leito. 
Tabela 3 - Planejamento Composto Central para avaliação dos parâmetros e resultados de porosidade.

\begin{tabular}{|c|c|c|c|c|c|c|c|}
\hline Simulação & $\begin{array}{c}\text { Módulo } \\
\text { Cisalhante } \\
{[\mathrm{Pa}]}\end{array}$ & $\begin{array}{l}\text { Coeficiente } \\
\text { Restituição }\end{array}$ & $\begin{array}{c}\text { Coeficiente } \\
\text { de Atrito } \\
\text { Estático }\end{array}$ & $\begin{array}{c}\text { Coeficiente de } \\
\text { Atrito de } \\
\text { Rolamento }\end{array}$ & $\begin{array}{c}\text { Energia de } \\
\text { Superfície } \\
{\left[\mathrm{J} / \mathrm{m}^{2}\right]}\end{array}$ & $\varepsilon_{D E M}$ & $\bar{\sigma}$ \\
\hline 1 & $-1,000$ & $-1,000$ & $-1,000$ & $-1,000$ & 1,000 & 0,519 & $-6,541$ \\
\hline 2 & $-1,000$ & $-1,000$ & $-1,000$ & 1,000 & $-1,000$ & 0,533 & $-3,425$ \\
\hline 3 & $-1,000$ & $-1,000$ & 1,000 & $-1,000$ & $-1,000$ & 0,558 & 2,182 \\
\hline 4 & $-1,000$ & $-1,000$ & 1,000 & 1,000 & 1,000 & 0,601 & 11,683 \\
\hline 5 & $-1,000$ & 1,000 & $-1,000$ & $-1,000$ & $-1,000$ & 0,484 & $-14,173$ \\
\hline 6 & $-1,000$ & 1,000 & $-1,000$ & 1,000 & 1,000 & 0,539 & $-2,024$ \\
\hline 7 & $-1,000$ & 1,000 & 1,000 & $-1,000$ & 1,000 & 0,544 & $-0,933$ \\
\hline 8 & $-1,000$ & 1,000 & 1,000 & 1,000 & $-1,000$ & 0,570 & 4,674 \\
\hline 9 & 1,000 & $-1,000$ & $-1,000$ & $-1,000$ & $-1,000$ & 0,501 & $-10,590$ \\
\hline 10 & 1,000 & $-1,000$ & $-1,000$ & 1,000 & 1,000 & 0,558 & 2,182 \\
\hline 11 & 1,000 & $-1,000$ & 1,000 & $-1,000$ & 1,000 & 0,574 & 5,609 \\
\hline 12 & 1,000 & $-1,000$ & 1,000 & 1,000 & $-1,000$ & 0,594 & 10,126 \\
\hline 13 & 1,000 & 1,000 & $-1,000$ & $-1,000$ & 1,000 & 0,504 & $-9,812$ \\
\hline 14 & 1,000 & 1,000 & $-1,000$ & 1,000 & $-1,000$ & 0,521 & $-6,073$ \\
\hline 15 & 1,000 & 1,000 & 1,000 & $-1,000$ & $-1,000$ & 0,537 & $-2,647$ \\
\hline 16 & 1,000 & 1,000 & 1,000 & 1,000 & 1,000 & 0,572 & 5,141 \\
\hline 17 & $-1,607$ & 0,000 & 0,000 & 0,000 & 0,000 & 0,567 & 4,051 \\
\hline 18 & 1,607 & 0,000 & 0,000 & 0,000 & 0,000 & 0,570 & 4,674 \\
\hline 19 & 0,000 & $-1,607$ & 0,000 & 0,000 & 0,000 & 0,580 & 7,011 \\
\hline 20 & 0,000 & 1,607 & 0,000 & 0,000 & 0,000 & 0,535 & $-2,958$ \\
\hline 21 & 0,000 & 0,000 & $-1,607$ & 0,000 & 0,000 & 0,466 & $-18,223$ \\
\hline 22 & 0,000 & 0,000 & 1,607 & 0,000 & 0,000 & 0,571 & 4,986 \\
\hline 23 & 0,000 & 0,000 & 0,000 & $-1,607$ & 0,000 & 0,534 & $-3,270$ \\
\hline 24 & 0,000 & 0,000 & 0,000 & 1,607 & 0,000 & 0,575 & 5,920 \\
\hline 25 & 0,000 & 0,000 & 0,000 & 0,000 & $-1,607$ & 0,543 & $-1,245$ \\
\hline 26 & 0,000 & 0,000 & 0,000 & 0,000 & 1,607 & 0,562 & 2,961 \\
\hline 27 & 0,000 & 0,000 & 0,000 & 0,000 & 0,000 & 0,570 & 4,674 \\
\hline 28 & 0,000 & 0,000 & 0,000 & 0,000 & 0,000 & 0,561 & 2,805 \\
\hline
\end{tabular}

Figura 2 - Superfícies de Resposta para a porosidade em função de: (a) Coeficientes de atrito estático e Restituição; (b) Coeficiente de atrito estático e Energia de Superfície.
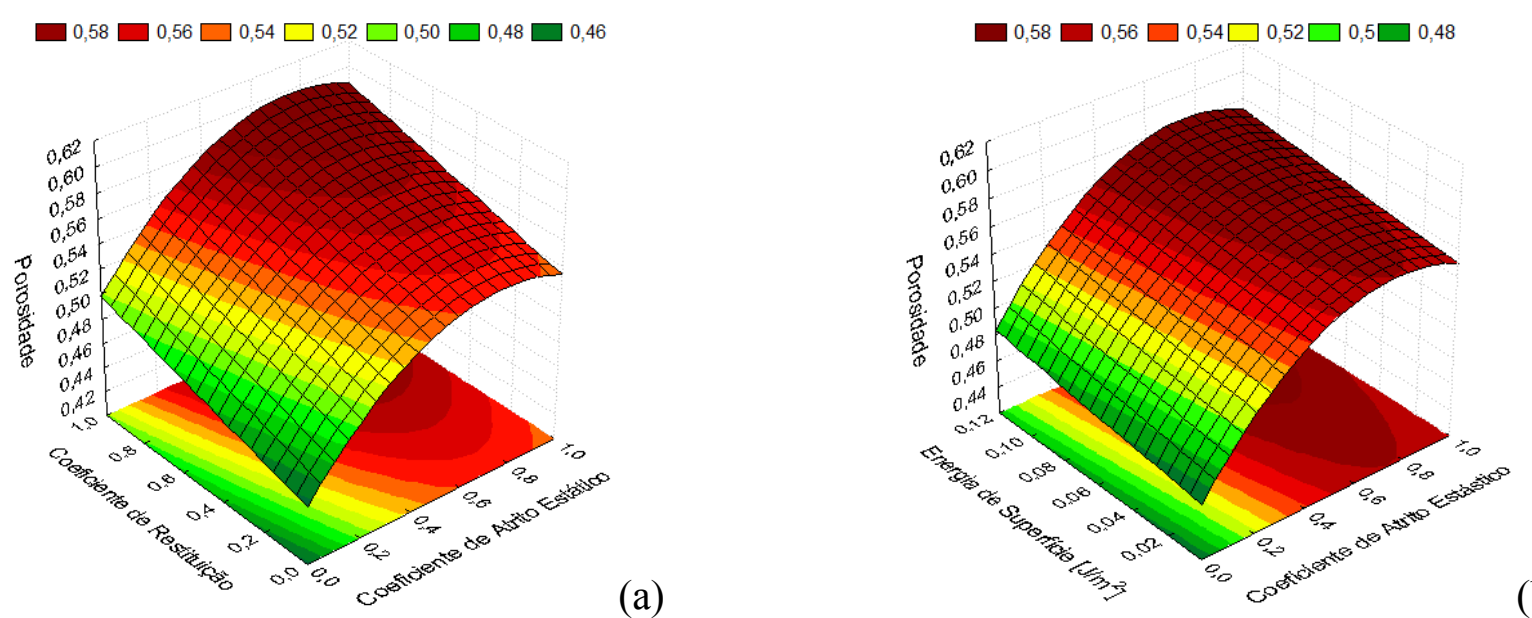

A Figura 3 apresenta o leito empacotado contendo as sementes de acerola pela simulação 7 (Tabela 3), que foi a condição simulada que mais se aproximou da condição experimental da porosidade $(\varepsilon=0,548)$. A Figura 4 mostra a porosidade em função da altura do leito. Os valores de porosidade aumentam significativamente em alturas próximas à base do 
cone. Isto ocorre devido à diminuição gradual do raio do cone, tornando mais significante o efeito de parede. Esse efeito deixa de ser significante quando o diâmetro do recipiente é pelo menos 10 vezes maior que o da partícula.

Figura 3 - Simulação DEM do leito empacotado com sementes de acerola.

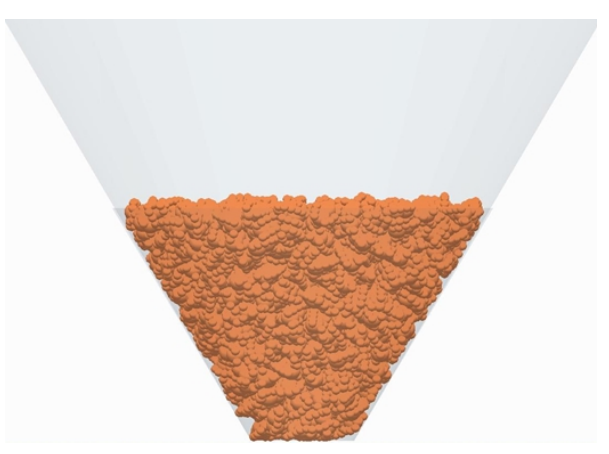

Figura 4 - Efeito de parede sobre a porosidade ao longo da altura do leito.

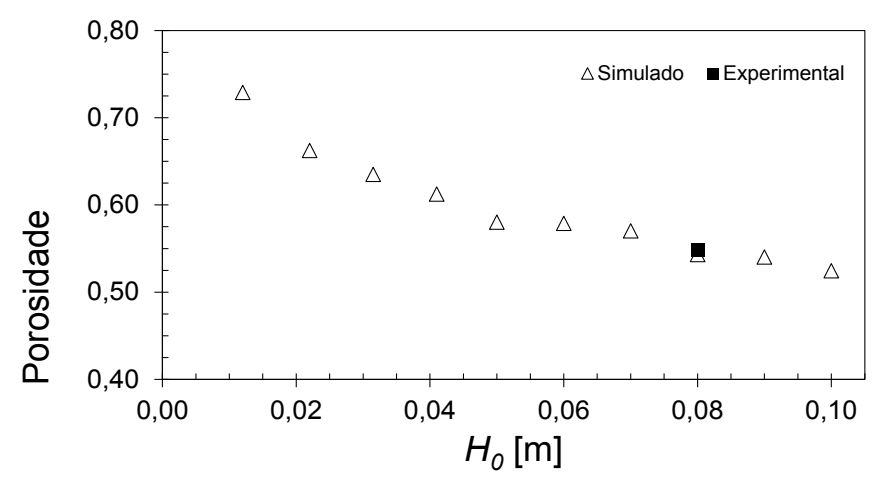

\section{CONCLUSÕES}

Neste trabalho, a metodologia de superfície de resposta foi empregada para avaliar o efeito dos parâmetros do modelo de força de contato sobre a porosidade de um leito contendo sementes de acerola. Observou-se que apenas o módulo cisalhante não influencia a resposta, e que o coeficiente de atrito estático é o que mais influencia a resposta. Comparando as simulações com o valor experimental de porosidade, foi possível encontrar o conjunto de parâmetros que representa adequadamente o comportamento experimental do empacotamento (simulação 7). Através das simulações, foi possível evidenciar que o sistema estudado apresenta efeito de parede, mostrando uma distribuição axial na porosidade no leito.

\section{REFERÊNCIAS}

BORTOLOTTI, C.T.; SANTOS, K.G.; FRANCISQUETTI, M.C.C.; DUARTE, C.R.; BARROZO, M.A.S. Hydrodynamic Study of a Mixture of West Indian Cherry Residue and Soybean Grains in a Spouted Bed. Can. J. Chem. Eng., v. 91, p. 1871-1880, 2013.

CLEARY, P.W. The effect of particle shape on simple shear flows. Powder Technology, v.179, p.144$163,2008$.

HERTZ, H. On the contact of elastic solids. J. reine und angewandte Mathematik, v. 92, p. 156-171, 1882.

MINDLIN, R.D. Compliance of elastic bodies in contact. J. Appl. Mech., v. 16, p. 259-268, 1949.

SANTOS, K.G., CAMPOS, A.V.P., OLIVEIRA, O.S., FERREIRA, L.V., FRANCISQUETTI, M.C.C., BARROZO, M.A.S. Simulação DEM do ângulo de repouso dinâmico de sementes de acerola: Estudo paramétrico empregando a técnica de superfície de resposta. In: XX Congresso Brasileiro de Engenharia Química, Florianópolis. Anais do XX COBEQ, 2014.

YI , L. Y., DONG , K. J., ZOU , R. P. , YU, A. B. Coordination Number of the Packing of Ternary Mixtures of Spheres: DEM Simulations versus Measurements, Ind. Eng. Chem. Res., v.50, p. 8773-8785, 2011. 